\section{RMD Open}

Rheumatic \&

Musculoskeletal Diseases

\title{
Initial validation and results of the Symptoms in Persons At Risk of Rheumatoid Arthritis (SPARRA) questionnaire: a EULAR project
}

\author{
Marian $\mathrm{H}$ van Beers-Tas, ${ }^{1,2}$ Marieke $\mathrm{M}$ ter Wee, ${ }^{3,4}$ Lilian $\mathrm{H}$ van Tuyl, ${ }^{3}$ Bertha Maat, ${ }^{1}$ \\ Wijnanda Hoogland, ${ }^{1}$ Aase $\mathrm{H}$ Hensvold, ${ }^{5}$ Anca I Catrina ${ }^{5}$ Erika Mosor, ${ }^{6,7}$ \\ Tanja A Stamm, ${ }^{6}$ Axel Finckh, ${ }^{8,9}$ Delphine S Courvoisier, ${ }^{8,9}$ Andrew Filer, ${ }^{10,11}$ \\ Ilfita Sahbudin, ${ }^{10,11}$ Rebecca J Stack, ${ }^{12}$ Karim Raza, ${ }^{13}$ Dirkjan van Schaardenburg ${ }^{1,2}$
}

To cite: van Beers-Tas MH, ter Wee MM, van Tuyl LH, et al. Initial validation and results of the Symptoms in Persons At Risk of Rheumatoid Arthritis (SPARRA) questionnaire: a EULAR project. RMD Open 2018;4:e000641. doi:10.1136/ rmdopen-2017-000641

- Prepublication history and additional material for this paper are available online. To view these files, please visit the journal online (http://dx.doi. org/10.1136/rmdopen-2017000641).

The manuscript was based on work previously presented at both the ACR conference 2016 and EULAR conference 2017 and published as abstracts: http://acrabstracts.org/abstract/ first-report-of-symptomsusing-the-symptoms-inpersons-at-risk-of-rheumatoidarthritis-sparra-questionnaire/; http://scientific.sparx-ip.net/ archiveeular/?searchfor=spar ra20questionnaire\&view $=1 \& \mathrm{c}=$ a\&item=2017FRI0113.

Received 24 December 2017 Revised 6 April 2018 Accepted 11 April 2018

Check for updates

For numbered affiliations see end of article.

Correspondence to Dr Marian H van Beers-Tas; m.h.tas@amc.uva.nl

\section{ABSTRACT}

Objectives To describe the development and assess the psychometric properties of the novel 'Symptoms in Persons At Risk of Rheumatoid Arthritis' (SPARRA) questionnaire in individuals at risk of rheumatoid arthritis (RA) and to quantify their symptoms.

Methods The questionnaire items were derived from a qualitative study in patients with seropositive arthralgia. The questionnaire was administered to 219 individuals at risk of RA on the basis of symptoms or autoantibody positivity: $74 \%$ rheumatoid factor and/or anticitrullinated protein antibodies positive, $26 \%$ seronegative. Validity, reliability and responsiveness were assessed. Eighteen first degree relatives (FDR) of patients with RA were used for comparison.

Results Face and content validity were high. The testretest showed good agreement and reliability (1 week and 6 months). Overall, construct validity was low to moderate, with higher values for concurrent validity, suggesting that some questions reflect symptom content not captured with regular Visual Analogue Scale pain/well-being. Responsiveness was low (small subgroup). Finally, the burden of symptoms in both seronegative and seropositive at risk individuals was high, with pain, stiffness and fatigue being the most common ones with a major impact on daily functioning. The FDR cohort (mostly healthy individuals) showed a lower burden of symptoms; however, the distribution of symptoms was similar.

Conclusions The SPARRA questionnaire has good psychometric properties and can add information to currently available clinical measures in individuals at risk of RA. The studied group had a high burden and impact of symptoms. Future studies should evaluate whether SPARRA data can improve the prediction of RA in at risk individuals.

\section{INTRODUCTION}

A range of symptoms can be present in individuals at risk of rheumatoid arthritis (RA). These individuals are usually defined based on either autoantibody positivity or symptoms. In seropositive at risk persons, symptoms usually

\section{Key messages}

What is already known about this subject?

- A wide range of symptoms can be present in individuals at risk of rheumatoid arthritis (RA), including extra-articular symptoms.

- These symptoms can be severe and disabling.

What does this study add?

- This study used data from qualitative focus interviews to quantify symptoms in individuals at risk of RA.

- The 'Symptoms in Persons At Risk of Rheumatoid Arthritis' (SPARRA) questionnaire provides information on location, timing and severity of these symptoms in a large international sample of individuals at risk of developing $\mathrm{RA}$.

How might this impact on clinical practice?

- The SPARRA questionnaire can be used to document symptoms in studies of persons at risk of RA.

occur later than seropositivity. ${ }^{12}$ However, information on location, timing and severity of symptoms is still largely lacking. ${ }^{3}$

Symptoms such as joint pain, swelling and morning stiffness represent key elements in the diagnosis of RA. Clinicians have tried to use these and other symptoms to identify those at risk of RA before they fulfil classification criteria for this condition. ${ }^{4}$ A European League Against Rheumatism (EULAR) taskforce recently outlined symptoms that were deemed most relevant in differentiating those at risk of developing RA (also known as 'clinically suspect arthralgia' (CSA) ${ }^{5}$ ) from other patients with non-specific joint symptoms. ${ }^{6}$ The criteria set for CSA were based 
on expert opinion and have shown value in predicting arthritis. $^{7}$

Qualitative research in individuals at risk of RA provided a different starting point to evaluate symptoms using the experience of the affected persons to understand the range of their symptomatology. With this approach, multiple focus group interviews were performed in patients with seropositive arthralgia. ${ }^{8-10}$ Besides symptoms originating from the joints, additional extra-articular themes emerged such as fatigue, distress and loss of motor control, with a reported major impact on daily functioning. The presumed impact of such early symptoms is underscored by increased sick leave and medical ambulatory costs long before diagnosis of RA. ${ }^{11} 12$

The 'Symptoms in Persons At Risk of Rheumatoid Arthritis' (SPARRA) questionnaire was developed based on data from our previous qualitative study. ${ }^{9}$ The aim of the present study was to describe the developmental process and test the psychometric properties of the SPARRA questionnaire in an international convenience sample of individuals at risk of RA (both autoantibody positive and negative) and to quantify and describe their symptoms based on this questionnaire.

\section{PATIENTS AND METHODS}

\section{The development of the SPARRA questionnaire}

The content of the SPARRA questionnaire is based on focus group interviews in 15 patients with seropositive arthralgia and 11 patients with early RA with whom initial symptoms prior to the diagnosis of RA were explored. ${ }^{9}$ These semistructured interviews were conducted to explore perceptions of symptoms, impact of symptoms and reactions to symptoms and continued until thematic saturation was reached. The content of the questionnaire was also informed by a previous review of the literature related to the earliest symptoms of $\mathrm{RA}^{4}$ and prior research describing domains that were deemed important in predictive algorithms in these at-risk individuals. ${ }^{1314}$ The emerging themes were grouped and the most noteworthy and frequently occurring categories were selected. Feedback from the study team (two rheumatologists, one epidemiologist, one expert on psychological testing and two research patient partners) was used to discuss which symptoms to be captured within the questionnaire, discuss realistic timeframes for symptom duration and the number/format of answer categories for severity and impact of the symptoms, taking into account reported variation in each of the domains by individuals from the focus group interviews. Afterwards, four rheumatologists from different countries, working in the field of the at-risk phase of RA but who were not otherwise involved in the project, gave their feedback on the questionnaire. The final questionnaire included 13 symptoms, for which severity and impact were described from none to severe and no to high, respectively. Additional questions were aimed at capturing location and pattern of joint pain (if present) and the presence of morning stiffness. Recently, data on symptoms in the at-risk phase of RA appeared in literature from two other cohorts and one review. These studies contain items on functional limitations, such as difficulty making a fist, which are possibly additive to the SPARRA questionnaire which only contains the following symptoms on function: 'weakness or loss of motor control' and 'impact of symptoms on daily functioning'. ${ }^{15-17}$ The questionnaire's design and content was thereafter discussed with patient research partners from both Amsterdam and Birmingham to assess face validity which led to only minor comments and small modifications.

Subsequently, the questionnaire was translated from English into Dutch, Swedish, German and French by at least one native speaker. These native speakers were part of the study teams at the different centres and had knowledge of research in individuals at risk of developing RA, but were not part of the study team that performed the focus interviews leading to the questionnaire development. Thereafter, another researcher from that study team translated it back to English, blinded for the original wording of the items, to complete the formal forward-backward approach as presented by the WHO (steps 1 and 2, except for the fact that back-translation was not performed by a whole expert panel). ${ }^{18}$ All inconsistencies were resolved in collaboration with a member of the original focus group interview team ( $\mathrm{LvT}$ ), by referring to the original wording in the focus groups. Cross-cultural adaptations were made taking into account cultural aspects of presenting joint symptoms within the different countries. ${ }^{19}$ As a preliminary pilot test, the Dutch prefinal version of the questionnaire was administered to 30 seropositive individuals with arthralgia from the Netherlands, which did not change the questionnaire (WHO steps 3 and 4, no cognitive interviewing was performed) ${ }^{18}$ To pre-empt any missing symptoms individuals had the possibility of adding up to two additional symptoms that they thought were relevant. See table 1 for an outline of the questionnaire (complete questionnaire and translated versions presented as online supplementary figures 1-5).

\section{Study participants}

To test the psychometric properties of the SPARRA questionnaire, individuals at risk of developing RA defined as individuals with RA-specific autoantibodies (anticitrullinated protein antibodies (ACPA) and/or rheumatoid factor (RF) ) or the presence of relevant symptoms (ie, individuals with CSA based on clinical expertise in the different centres with or without RA-specific antibodies) were selected from four European centres: Reade, Amsterdam ( $\mathrm{n}=125)$ (further called the Netherlands), Sandwell and West Birmingham Hospitals and the University Hospitals Birmingham (n=69) (UK), Karolinska University Hospital $(\mathrm{n}=15)$ (Sweden) and the Medical University of Vienna $(n=10)$ (Austria). We have used an international sample of patients at risk for RA, mainly containing consecutive cohort patients (Netherlands, UK, Sweden, in total 88\%) and complemented by a convenience sample from Austria and Switzerland. Please note that in Austria, patients were recruited from 
Table 1 Outline of the SPARRA questionnaire

Per symptom (see right), the following questions are asked:

1. Over the past month how many days of the month have you had (symptom)?

2. Over the past month how much (symptom) have you had?

3. What impact has this (symptom) had on your ability to carry out daily activities (eg, work, household chores, childcare, social activities)?

4. Where did you feel the [symptoms 1 to 9]

1. 0 days, $1-5$ days, $6-16$ days, $16-30$ days

2. None, mild, moderate, severe

3. No impact, small impact, moderate impact, large impact

4. Hand (one or both), arm (one or both), foot (one or both), leg (one or both)
Answer categories:

1. Joint pain (symptoms)

2. Joint swelling

3. Joint stiffness

4. Burning sensations

5. Tingling sensations

6. Numbness

7. Changes in skin colour over joints

8. Muscle cramps

9. Weakness or loss of strength

10. Fatigue

11. Emotional distress

12. Concentration difficulties

13. Sleep problems

\section{Additional questions:}

- Description of joint pain (burning, sharp/stabbing, aching, other)

- Movement of joint pain (no, arms to legs, legs to arms, one side to the other)

- Presence of morning stiffness (no, <1 hour, 1-2 hours, all morning)

Rate the average joint pain over the last month in different body areas:

- Answer categories: no pain, mild moderate, severe

- Fingers (left/right), wrist (left/right), elbow (left/right), shoulder (left/right), hip (left/right), knee (left/right), ankle (left/right), toes (left/right), neck, back

What is the pattern of symptom development since the time they first began (see patterns on the right; patients could also draw a pattern themselves)
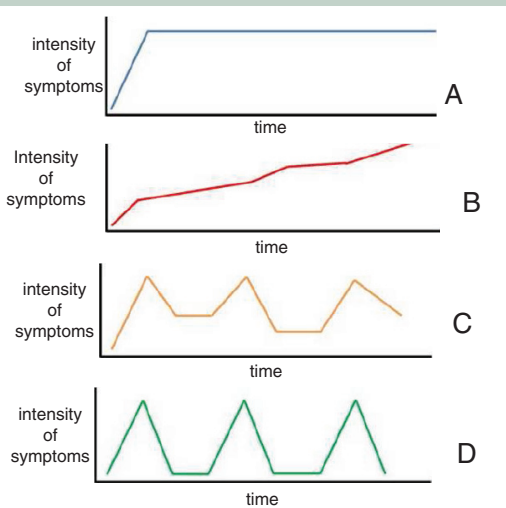

Note: full questionnaire (with translations) added as online supplementary material.

SPARRA, Symptoms in Persons At Risk for Rheumatoid Arthritis.

their 'pre-arthritis' cohort and by additionally searching through their clinical database for patients who were ACPA-positive and/or RF-positive without a diagnosis of arthritis. In Switzerland, individuals could complete a SPARRA questionnaire at any time when they visited for a yearly cohort follow-up. All cohorts were set up to characterise individuals at risk of developing RA and included individuals without prior arthritis (see online supplementary table 1). ${ }^{2021}$ Arthritis was assessed clinically (by a rheumatologist in all cohorts) by presence of at least one swollen joint: no confirmation by ultrasonography or MRI was used. A cohort of 18 first degree relatives (FDR) of patients with RA from the University Hospital of Geneva (Switzerland) was used as comparison, since they also represent a group of individuals at risk of developing RA which the SPARRA questionnaire is aimed at. This cohort was dealt with separately, since the individuals were recruited based on the fact that they were FDR and not because of symptoms or antibodies and thus included mostly healthy individuals with an increased risk of RA. Individuals were included between November 2014 and December 2016.

\section{Study procedures}

At baseline, individuals completed the SPARRA questionnaire and had clinical data collected, including antibody status, total painful (tender joint count 44) and swollen joints (swollen joint count 44), family history, symptom duration, smoking status, Visual Analogue Scales (VAS, ranged 0-100) for pain, patient global assessment and fatigue. Detailed data on comorbidities and medication have not been consistently assessed across the cohorts for the present study. A subgroup of individuals had a follow-up measurement (test-retest) after 1 week and 6 months. Questionnaires completed at the time of or after clinical arthritis development (confirmed by a rheumatologist) were discarded for the current analysis. The study was approved by relevant Ethics Committees and all individuals gave written informed consent.

\section{Psychometric properties}

Content validity

Relevant medical articles on symptoms in the at risk phase of RA were compared with the questionnaire items to see if all relevant facets of the construct had been captured.

\section{Construct validity}

We performed correlations between baseline questionnaire items and clinical parameters that were deemed to be associated based on expert opinion (seven representatives from all centres). We divided these into items with a very close match (concurrent validity, eg, the item fatigue compared with VAS fatigue) and items with less close a match (construct validity). Individuals had to complete the questionnaire within 2 weeks of clinical measurements. 


\section{Agreement and reliability}

Test-retest analyses were performed in a subgroup of individuals that completed a second test within 7-14 days and/or after 6 months. The retest questionnaire was only sent after return of the first questionnaire. The analyses were performed in questions not containing time elements. Also, we described the scale reliability at baseline, that is, looking at how closely related the items in the questionnaire are as a group.

\section{Responsiveness}

This can only be tested in individuals with expected change in their disease status, in our case VAS scores over a time period of 6 months. No formal clinically relevant VAS score changes have been described in individuals at risk of developing RA. We chose a change of $11 \mathrm{~mm}$ as sometimes used in an adult rheumatology setting, ${ }^{22}$, and we measured a second arbitrary cut-off of $25 \mathrm{~mm}$, since the questionnaire items are on a 4-point scale. We only analysed questionnaire items that were concurrent with the VAS scores. The questionnaire had to be completed within 2 weeks from the clinical measurements.

\section{Statistical analyses}

Construct validity

Correlations were calculated using the Spearman's rank correlation coefficient. Statistical significance was set as a $\mathrm{p}$ value less than 0.05 . The VAS scores were used as continuous data. Interpretation: 0-0.30 small, 0.3-0.50 medium, $0.50-1$ large. ${ }^{23}$

\section{Test-retest agreement and reliability}

The percentage of agreement in the questionnaire items was given for questions on symptom severity and impact, and the Cohen's weighted kappa (reliability) was measured. ${ }^{24}$ Interpretation: $<0$ no, $0-0.20$ slight, $0.21-0.40$ fair, 0.41-0.60 moderate, $0.61-0.80$ substantial and $0.81-1$ almost perfect agreement. ${ }^{25}{ }^{26}$ Scale reliability was described with the Cronbach's alpha.

\section{Responsiveness}

The Wilcoxon signed-rank test was used to analyse significant differences between questionnaire items over the 6-month period, taking $\mathrm{p}<0.05$ as significance level.

\section{Handling missing data}

All questions in the questionnaire follow the same pattern (table 1). If subquestions ' $a$ ' (duration of the symptom over the past month) were missing and 'b-d' were also missing, then ' $a$ ' was set as 0 days. Equally, if ' $a$ ' was set as 0 days, then ' $b-d$ ' were set as none, no impact and not filled in, respectively. Instead, if ' $b-d$ ' were filled out while ' $a$ ' was missing, then we assumed the worst case scenario and set 'a' as 16-30 days.

All analyses were performed with SPSS V.21 (IBM, Armonk, New York, USA), except for the Cohen's weighted kappa's which were computed using Stata V.13.1 (Stata 2013, College Station, Texas, USA).
Frequency and impact of symptoms in the SPARRA questionnaire

Finally, we analysed data from individuals who were ACPA-positive (with or without RF), only RF-positive and those included in the cohort due to specific symptoms (seronegative CSA). Percentages of symptom duration in the last month (dichotomised to $0-15$ days and 16 or more days), severity (none/mild vs moderate/severe) and impact (no/small vs moderate/large) were given for these groups and information on joint pain location and patterns was described.

\section{RESULTS}

\section{Study population}

Two hundred and nineteen individuals completed the SPARRA questionnaire in 4 European centres, with 18 FDR of patients with RA as comparison (online supplementary table 2). Half of the individuals (excluding the FDR) were ACPA-positive (with or without RF), $24 \%$ were only RF-positive and 26\% were seronegative with CSA (table 2). The mean age of these study participants was 49 years (SD 13.2) and the median duration of symptoms was 20 months (25th-75th percentile 8-56).

\section{Content validity}

The items in the questionnaire represent symptoms that are important for individuals at risk of RA. ${ }^{13} 1^{27-29}$ Literature search did not identify any additional symptoms describing the at risk phase, except for self-reported functional limitation as part of a tool to detect early inflammatory arthritis ${ }^{30}$ and difficulty in making a fist at physical examination in individuals with CSA. ${ }^{6}$ In addition to the seropositive individuals, we also selected seronegative individuals with CSA and FDRs to achieve a good representation of the at risk population. To make sure that no key symptoms were omitted, in addition to the 13 predefined symptoms, individuals had the option of adding two symptoms. Forty-three out of the 219 individuals used this option (of whom 14 reported two options). Themes (reported at least twice) were: pain/inflammation around tendons or myalgia $(n=7)$, pain only while using the joint $(n=4)$, dry eyes $(n=4)$, functional limitations $(n=2)$, itching skin spots $(n=2)$ and swelling in the groin or legs $(n=2)$. However, many of them did not describe new symptoms $(\mathrm{n}=16$ alternative diagnosis that they felt explained their symptoms such as osteoarthritis or hernia, $\mathrm{n}=5$ explanatory description, $\mathrm{n}=2$ unclear).

\section{Construct validity (relation to clinical parameters)}

Analyses were performed in 208 individuals, since 11 did not have clinical data collected within 2 weeks from the baseline questionnaire. Overall, the correlation between questionnaire items and clinical parameters (VAS pain, VAS global assessment and VAS fatigue) was medium to large with Spearman coefficients ranging from 0.38 to 0.63 (online supplementary table 3 , all statistically significant). Correlations were higher when strictly looking at concurrent validity $(0.58-0.63)$. The percentage of 
Table 2 Baseline characteristics $(n=219)$

\begin{tabular}{|c|c|c|c|c|}
\hline Variable & $\begin{array}{l}\text { ACPA-positive } \\
\text { individuals* }(n=109)\end{array}$ & $\begin{array}{l}\text { RF-positive } \\
\text { individuals* } \\
(n=53)\end{array}$ & $\begin{array}{l}\text { Seronegative } \\
\text { individuals with CSA } \\
(n=57)\end{array}$ & $\begin{array}{l}\text { FDRs of patients with } \\
\text { RA† } \\
(n=18)\end{array}$ \\
\hline Age $\ddagger$ & 49 (12.9) & 54 (13.2) & 45 (12.6) & $57(9.5)$ \\
\hline Females (\%) & 72 & 64 & 72 & 89 \\
\hline $\begin{array}{l}\text { Symptom duration } \\
\text { (months)§ }\end{array}$ & $23(10-52)$ & $30(12-60)$ & $11(4-39)$ & $22(7-51)$ \\
\hline $\begin{array}{l}\text { Tender joint count (44 } \\
\text { joints) }\end{array}$ & $0(0-2)$ & $0(0-2)$ & $2(0-7)$ & $1(0-3)$ \\
\hline VAS pain $(\mathrm{mm}) \S$ & $18(2-56)$ & $27(3-47)$ & $56(34-71)$ & ND \\
\hline $\begin{array}{l}\text { VAS patient global } \\
\text { assessment }(\mathrm{mm}) \S\end{array}$ & $28(3-56)$ & $22(0-49)$ & 48 (25-69) & ND \\
\hline VAS fatigue $(\mathrm{mm}) \S$ & $50(9-80)$ & $33(6-59)$ & $65(40-82)$ & ND \\
\hline Current smoking (\%) & 24 & 15 & 25 & 17 \\
\hline FDR with RA (\%)§ & 29 & 21 & 28 & 100 \\
\hline
\end{tabular}

*ACPA-positive individuals: with or without RF positivity, RF-positive individuals: only RF-positive.

†FDRs of patients from Switzerland with RA were used as comparison cohort.

$\ddagger$ Mean (SD), all other continuous variables mentioned as median (25th-75th percentile).

§Missing values; $2 \%$ for VAS global and family history, $3 \%$ for VAS pain, $4 \%$ for VAS fatigue, $6 \%$ for symptom duration, one individual for RF (marked as ACPA positive only now).

Netherlands: $A C P A+n=71 ; R F+n=40$; seronegative $n=14$.

UK: ACPA $+n=21 ; R F+n=8$; seronegative $n=40$.

Sweden: $A C P A+n=15 ; R F+n=0$; seronegative $n=0$.

Austria: $A C P A+n=2 ; R F+n=5$; seronegative $n=3$.

Switzerland: ACPA+ $n=6$; $R F+n=1$; seronegative $n=11$.

ACPA, anticitrullinated protein antibodies; CSA, clinically suspect arthralgia; FDR, first degree relative; ND, not done; RA, rheumatoid

arthritis; RF, rheumatoid factor; VAS: Visual Analogue Scale.

missing values in the questionnaire items was $1 \%(15$ missing questions were set on the worst case scenario, 91 were set on no symptoms) and in the clinical parameters $3 \%$.

\section{Test-retest reliability and agreement and scale reliability}

Analyses after 1 week were performed in 51 individuals (20 ACPA+, $26 \mathrm{RF}+, 5$ seronegative) and after 6 months in 90 individuals (37 ACPA+, $30 \mathrm{RF}+, 23$ seronegative). The median time difference in the latter 90 individuals between the questionnaires was 6 months (25th-75th percentiles: 5-10). Thirty-eight individuals had a retest after both 1 week and 6 months. Overall, the test-retest agreement for the questions was good to excellent $(88 \%-$ $98 \%$ ) after 1 week, and good reliability with Cohen's weighted kappa's between 0.60 and 0.90 was found (table 3 ). After 6 months, the agreement was $73 \%-91 \%$, with lower overall kappa's between 0.09 and 0.62 . Less than $1 \%$ of the data was missing (14 missing questions were set on the worst case scenario, 30 were set on no symptoms). Subgroup analysis was not feasible due to low numbers. The Cronbach's alpha for all items on duration, severity and impact was $0.859,0.874$ and 0.908 , respectively ( 0.958 if all items were combined).

\section{Responsiveness}

Seventy-four individuals had clinical and questionnaire data after a follow-up of 6 months and within 2 weeks apart. Of these, 31 individuals had a VAS pain change of $11 \mathrm{~mm}$ and 12 individuals a VAS pain change of $25 \mathrm{~mm}$; equivalent data for VAS fatigue were 32 individuals and 16 individuals (17\% missing VAS change scores). The Wilcoxon signed-rank test for the questionnaire items joint pain severity and impact was non-significant when using both cut-off points for VAS pain $(p=0.46$ and $\mathrm{p}=0.24$, respectively for cut-off $11 \mathrm{~mm}$ and both $\mathrm{p}=0.43$ for cut-off $25 \mathrm{~mm}$ ). Also, no statistically significant differences were found for fatigue severity/impact for the VAS fatigue ( $p=0.21$ and $p=0.63$, respectively, for cut-off $11 \mathrm{~mm}$, and $\mathrm{p}=0.11$ and $\mathrm{p}=0.15$, respectively, for cut-off $25 \mathrm{~mm})$.

\section{Frequency and impact of symptoms}

The frequency of symptoms and their impact in the individuals at risk of RA was high (table 4). Overall, presence of symptoms was reported more often by the seronegative individuals with CSA, followed by the ACPA-positive and then the RF-positive group (except for fatigue which occurred more often in ACPA-positive individuals). In all three groups, the percentage of individuals with symptoms at least 16 days in the past month was highest for joint pain $(37 \%-72 \%)$, joint stiffness $(34 \%-68 \%)$, weakness or loss of strength $(21 \%-35 \%)$ and fatigue $(28 \%-$ $39 \%)$. The severity and impact were reported similarly across the three groups with the exception of burning 
Table 3 Test-retest analysis of the SPARRA questionnaire ( $\mathrm{n}=51$ after 1 week, $\mathrm{n}=90$ after 6 months)

\begin{tabular}{|c|c|c|c|c|c|c|}
\hline \multirow[b]{2}{*}{ Items } & \multicolumn{2}{|c|}{$\%$ agreement* } & \multicolumn{2}{|c|}{ Weighted kappa } & \multicolumn{2}{|c|}{$\begin{array}{l}\text { Percentage with the } \\
\text { symptom } \dagger\end{array}$} \\
\hline & 1 week & 6 months & 1 week & 6 months & 1 week & 6 months \\
\hline Joint pain: severity $\ddagger / i m p a c t \S$ & $84 / 92$ & $77 / 77$ & $0.60 / 0.78$ & $0.37 / 0.41$ & 75 & 80 \\
\hline Joint swelling: severity/impact & $95 / 94$ & $83 / 83$ & $0.80 / 0.77$ & $0.42 / 0.44$ & 28 & 37 \\
\hline Joint stiffness: severity/impact & $88 / 93$ & $79 / 81$ & $0.71 / 0.78$ & $0.47 / 0.45$ & 69 & 74 \\
\hline Burning sensations: severity/impact & $90 / 92$ & $81 / 83$ & $0.64 / 0.61$ & $0.43 / 0.42$ & 20 & 33 \\
\hline Tingling sensations: severity/impact & $90 / 93$ & $85 / 84$ & $0.62 / 0.64$ & $0.53 / 0.35$ & 41 & 40 \\
\hline Numbness: severity/impact & $90 / 94$ & $83 / 85$ & $0.53 / 0.67$ & $0.27 / 0.27$ & 26 & 27 \\
\hline $\begin{array}{l}\text { Changes in skin colour: severity/ } \\
\text { impact }\end{array}$ & $96 / 98$ & $83 / 91$ & $0.67 / 0.71$ & $0.09 / 0.20$ & 20 & 20 \\
\hline Muscle cramps: severity/impact & $92 / 95$ & $84 / 87$ & $0.63 / 0.71$ & $0.43 / 0.39$ & 28 & 36 \\
\hline $\begin{array}{l}\text { Weakness or loss of strength: } \\
\text { severity/impact }\end{array}$ & $92 / 93$ & $78 / 76$ & $0.81 / 0.81$ & $0.43 / 0.34$ & 57 & 56 \\
\hline Fatigue: severity/impact & 93/92 & $80 / 79$ & $0.81 / 0.82$ & $0.52 / 0.50$ & 69 & 63 \\
\hline Emotional distress: severity/impact & $93 / 95$ & $77 / 80$ & $0.78 / 0.83$ & $0.37 / 0.41$ & 47 & 39 \\
\hline $\begin{array}{l}\text { Concentration difficulties: severity/ } \\
\text { impact }\end{array}$ & $93 / 94$ & $86 / 87$ & $0.76 / 0.78$ & $0.58 / 0.58$ & 33 & 39 \\
\hline Sleep problems: severity/impact & $90 / 93$ & $81 / 83$ & $0.77 / 0.80$ & $0.54 / 0.54$ & 55 & 49 \\
\hline Left fingers/right fingers & $91 / 90$ & $78 / 73$ & $0.74 / 0.76$ & $0.41 / 0.32$ & $49 / 53$ & $61 / 53$ \\
\hline Left wrist/right wrist & 93/97 & $83 / 80$ & $0.75 / 0.90$ & $0.43 / 0.40$ & $35 / 28$ & $39 / 38$ \\
\hline Left elbow/right elbow & $96 / 95$ & $87 / 87$ & $0.79 / 0.78$ & $0.42 / 0.44$ & $20 / 22$ & $31 / 27$ \\
\hline Left shoulder/right shoulder & $94 / 93$ & $82 / 81$ & $0.81 / 0.77$ & $0.45 / 0.44$ & $39 / 37$ & $40 / 40$ \\
\hline Left hip/right hip & 95/95 & $88 / 83$ & $0.80 / 0.83$ & $0.52 / 0.40$ & $28 / 29$ & $29 / 33$ \\
\hline Left knee/right knee & $96 / 94$ & $82 / 82$ & $0.85 / 0.81$ & $0.34 / 0.46$ & $29 / 33$ & $42 / 43$ \\
\hline Left ankle/right ankle & $96 / 95$ & $85 / 91$ & $0.83 / 0.79$ & $0.47 / 0.62$ & $28 / 28$ & $27 / 22$ \\
\hline Left toes/right toes & $95 / 97$ & $87 / 88$ & $0.76 / 0.85$ & $0.49 / 0.56$ & $24 / 22$ & $29 / 32$ \\
\hline Neck/back & $90 / 90$ & $81 / 81$ & $0.73 / 0.74$ & $0.42 / 0.48$ & $55 / 55$ & $44 / 48$ \\
\hline
\end{tabular}

Interpretation of the weighted kappa: $0=$ only chance agreement, $\leq 0.20=$ poor, $0.21-0.40=$ fair, $0.41-0.60=$ moderate, $0.61-0.80=$ good, $0.81-1.00=e x c e l l e n t /$ perfect.

${ }^{*}$ Retest analysis per item of severity and impact of a symptom (severity: none, mild, moderate, severe; impact: no impact, small impact, moderate impact, large impact).

†Percentage of patients that had the symptom at least 1 day in the past month at week 1 and month 6 .

fFor each symptom answering the question: over the past month how much (symptom) have you had?

§For each symptom answering the question: what impact has this (symptom) had on the ability to carry out daily activities.

SPARRA, Symptoms in Persons At Risk of Rheumatoid Arthritis.

and tingling sensations and muscle cramps, which had a lower frequency in ACPA-positive individuals, but a higher impact and severity.

Joint pain was mostly reported in the fingers (ACPA+ 58\%, RF+ 52\%, seronegative with CSA 65\%); however, the percentage of neck and back pain was also high (ACPA+ 39\%, RF+ 46\%, seronegative with CSA $47 \%$ and ACPA $+50 \%, \mathrm{RF}+52 \%$, seronegative with CSA $57 \%$, respectively) (figure 1). Usually, this joint pain was described as aching, symmetric and only one-third reported them as mild. The location of joint pain had a similar distribution across all groups.

Finally, we evaluated the pattern of joint pain in the period preceding the first questionnaire (table 1). Joint pain rapidly increasing and then remaining constant was reported by $9 \%$ (pattern A), joint pain gradually increasing over time by $16 \%$ (pattern B) and a more intermittent pattern by $53 \%$ (respectively $23 \%$ and $30 \%$ for in between periods without pain (D) and symptoms coming and going but always some pain present $(\mathrm{C})$ ) (see table 4 for classification by antibody positivity or negativity). Fourteen individuals $(6 \%)$ had missing data. The remainder of the individuals chose the option of either drawing a pattern themselves or describing it. Of those, one was similar to A, five similar to $\mathrm{C}$ and six to D. Of the remaining 25,6 had no symptoms, 11 had a peak in the beginning and then declining symptoms (mostly to 0 ), 3 had a combination of the intermittent patterns, 1 reported an intermittent pattern with no remaining symptoms afterwards, 3 were unclear and the last individual filled in both $\mathrm{A}$ and $\mathrm{C}$. 
Table 4 Duration, severity and impact of the SPARRA questionnaire items $(n=237)$ and joint pain patterns $(n=223)$

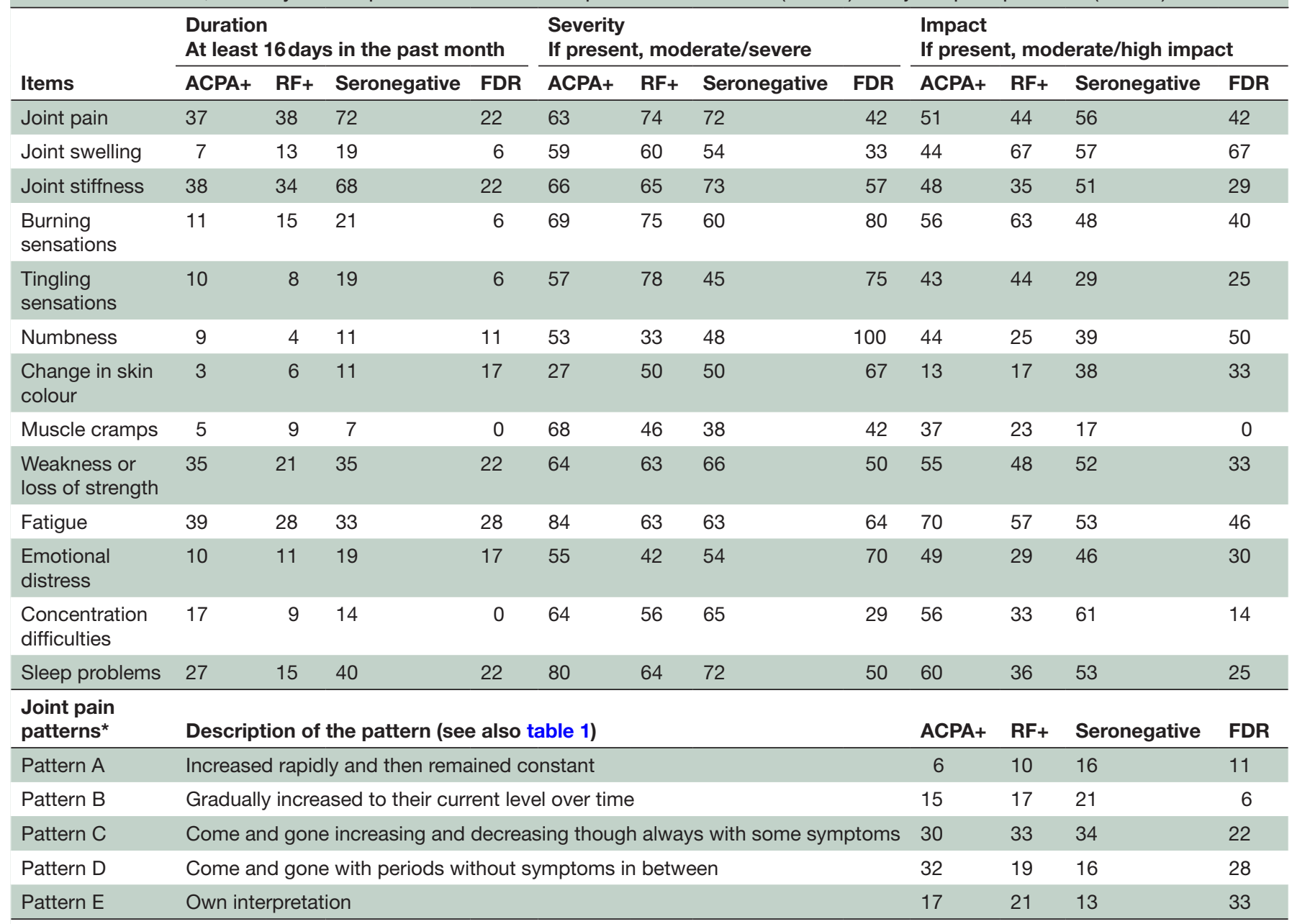

Data expressed as percentages. Note that the ACPA-positive group also includes RF-positive individuals, and all RF positive individuals are ACPA-negative.

${ }^{*}$ Missing data in 14 individuals.

ACPA, anticitrullinated protein antibodies; FDR, first degree relatives; RF, rheumatoid factor; SPARRA, Symptoms in Persons At Risk of Rheumatoid Arthritis.

\section{Comparison with FDR of patients with RA}

We compared the questionnaire data with 18 FDR of patients with RA. Missing data in this cohort were very low (1 missing question and 13 questions answered with two options were set on the worst case). The prevalence of symptoms was lower, except for numbness and change in skin colour (table 4). The location of joint pain was similar for these individuals (figure 1), just like joint pain patterns $\mathrm{A}, \mathrm{C}$ and $\mathrm{D}(11 \%, 22 \%$ and $28 \%$, respectively). The percentage of pattern B was shifted towards the open option where individuals could fill in a pattern themselves (B in 5.6\% and other in 33\%). Of the latter six individuals, three had no symptoms, two had a peak in the beginning and then no pain and the last can be set as D.

\section{DISCUSSION}

The SPARRA questionnaire has good psychometric properties. The data show a high burden, severity and impact of symptoms in individuals at risk of developing RA.
Evaluation of a complete set of symptoms that might be predictive for RA development is a challenge in the setting of prospective cohort studies, since questions need to be predetermined and are usually based on classification criteria. Using the SPARRA items derived directly from ACPA-positive symptomatic at-risk individuals gave the opportunity to gain more insight into these symptoms.

Questionnaires including symptoms have not yet been reported in cohorts researching individuals at risk of RA. Some use generic tools addressing functional limitations rather than specific symptoms, for example, the 36-item Short Form survey and EuroQol five dimensions questionnaire, which have shown their relevance in patients with RA and other musculoskeletal disease, but not yet in the at risk phase. ${ }^{31-33}$ Recently, the use of the Health Assessment Questionnaire (HAQ) has been described in individuals with CSA and it was shown that overall a low score (median 0.5) was present, but a higher score seemed to correlate with inflammation on MRI and arthritis development. ${ }^{17}$ 


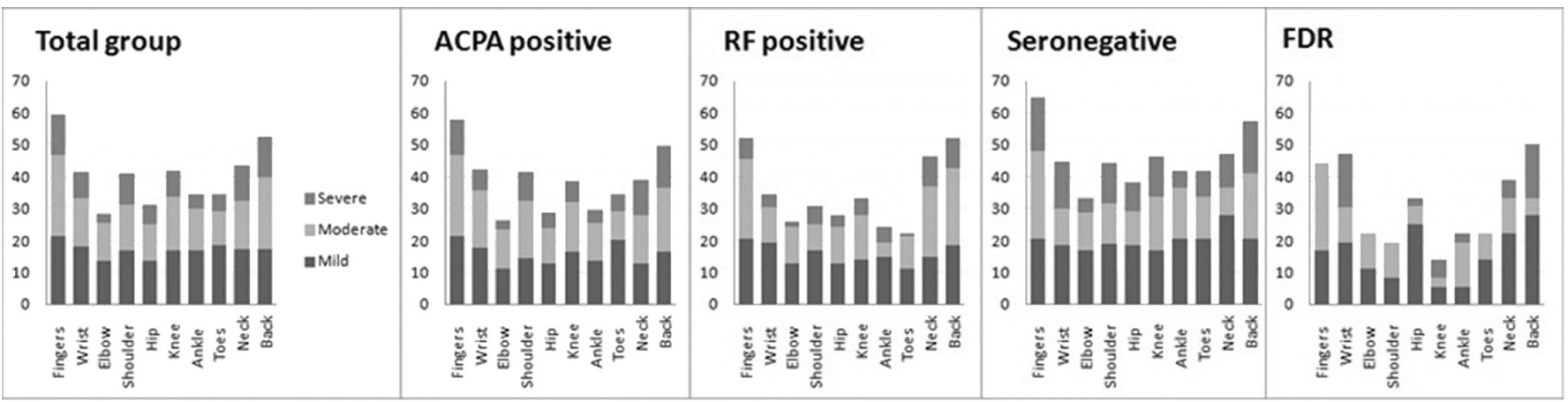

Figure 1 Joint pain location and severity. Reported answers to the following question: 'how much pain did you have on average over the last month in mentioned body areas?' Levels of pain ranging from no to severe, expressed as mean percentage of the left and right side of the body. Total group: ACPA and/or RF-positive individuals combined with seronegative individuals. FDR of patients with RA presented as comparison group. ACPA, anticitrullinated protein antibodies; FDR, first degree relatives; $\mathrm{RA}$, rheumatoid arthritis; RF, rheumatoid factor.

Construct validity and responsiveness of the SPARRA questionnaire were moderate and non-significant (respectively) in this study. The moderate correlation between clinically used VAS scores with questionnaire items can partly be explained by the fact that VAS scores measure symptoms from the past week compared with SPARRA questionnaire items measuring symptoms during the last month. Alternatively, the questions may measure different elements and reflect symptom content not captured with regular VAS pain/global assessment. This would mean that the questionnaire adds information to currently available clinical measures in individuals at risk of RA. The low responsiveness in individuals with changing VAS pain and fatigue scores might relate to the fact that no formal cut-offs are described for this study population and responsiveness could only be measured in a small subgroup. It could be that a change in the questionnaire items is not useful in follow-up of individuals at risk of RA; however, future evaluation in a larger population in a longitudinal setting is necessary.

The data showed that besides the expected joint symptoms, the burden of general and nervous system-related symptoms such as burning and tingling sensations, numbness and fatigue was high, especially among the ACPA-positive individuals. An explanation could be the presence of a more general subclinical inflammation as was suggested by MRI and PET studies ${ }^{29} 34$ as well as an early involvement, prior to subclinical inflammation, of the neurosystem in ACPA-positive individuals as both in-vivo and in-vitro studies have suggested. ${ }^{35}{ }^{36}$ Higher scores in joint pain and joint stiffness in the seronegative individuals might be a consequence of the fact that these individuals were included mainly based on the presence of symptoms. This was underscored by a recent cohort study in which a difference was shown between the symptomatic phase preceding ACPA-positive and ACPA-negative RA, and seronegative individuals had more symptoms at baseline. ${ }^{15} \mathrm{~A}$ lower burden of symptoms in the cohort of FDR of patients with RA was expected as these individuals mostly were without symptoms or antibodies at completion of the baseline SPARRA questionnaire. A similar joint pain location and pattern in the FDR's as compared with the seropositive arthralgia group may reflect their increased risk for RA. ${ }^{16}$

For missing data imputation in this study, we decided to use a worst case scenario approach. We also checked whether using a best case scenario changed the results, which was not the case (data not shown).

A limitation of the study may be selection bias caused by partly using a convenience sample in which non-response and the associated reasons are lacking. This may have led to overestimation of the burden of disease, since individuals with more symptoms may be more willing to complete the questionnaire. However, since the (heterogeneous) study population was taken from a set of individuals found in daily practice in secondary care, we expect that the results from the study can be generalised to other secondary care practices. Also, $88 \%$ of individuals were assessed in a consecutive manner. Another limitation is the fact that comorbidities may influence the reported symptoms and data on comorbidities were not collected for the present study.

The predictive value of the questionnaire items (possibly combined with items of the HAQ) for developing RA requires further investigation. This can hopefully guide item reduction of the questionnaire in the future. Furthermore, responsiveness could also be investigated in medication trials for preventing RA and a longitudinal design would be helpful to assess its use.

In conclusion, this study provides evidence of good psychometric properties of the SPARRA questionnaire, except for moderate construct validity and low responsiveness. In individuals at risk of RA, symptoms are frequent and severe and have a high impact. Future studies are needed to evaluate whether data from the SPARRA questionnaire can help to improve the prediction of RA.

\section{Author affiliations}

${ }^{1}$ Amsterdam Rheumatology and Immunology Center, Reade, Amsterdam, The Netherlands

${ }^{2}$ Amsterdam Rheumatology and Immunology Center, Amsterdam Medical Center, Amsterdam, The Netherlands

${ }^{3}$ Amsterdam Rheumatology and Immunology Center, VU University Medical Center, Amsterdam, The Netherlands 
${ }^{4}$ Department of Epidemiology and Biostatistics, VU University Medical Center, Amsterdam, The Netherlands

${ }^{5}$ Karolinska University Hospital, Stockholm, Sweden

${ }^{6}$ Section for Outcomes Research, Center for Medical Statistics, Informatics and Intelligent Systems, Medical University of Vienna, Vienna, Austria

${ }^{7}$ Division of Rheumatology, Department of Internal Medicine III, Medical University of Vienna, Vienna, Austria

${ }^{8}$ Division of Rheumatology, University Hospital of Geneva, Geneva, Switzerland ${ }^{9}$ Department of Medical Specialties, University of Geneva, Geneva, Switzerland ${ }^{10}$ University Hospitals Birmingham NHS Foundation Trust, Birmingham, UK ${ }^{11}$ Institute of Inflammation and Ageing, University of Birmingham, Birmingham, UK ${ }^{12}$ Department of Psychology, Nottingham Trent University, Nottingham, UK ${ }^{13}$ Department of Rheumatology, Sandwell and West Birmingham Hospitals NHS Trust, Birmingham, UK

Acknowledgements We thank Jas Kaur, clinical trials practitioner at the rheumatology department of the City Hospital in Birmingham, for data entry of part of the questionnaires from the UK. Second, we also thank the EULAR standing committee of epidemiology and health services research for their support and members of the ESCIR study group for the risk factors for RA for useful discussions. Third, this report is independent research supported by the National Institute for Health Research/Wellcome Trust Clinical Research Facility at University Hospitals Birmingham NHS Foundation Trust. The Arthritis Research UK Rheumatoid Arthritis Pathogenesis Centre of Excellence is funded by Arthritis Research UK through grant number 20298. KR and AF were supported by the National Institute for Health Research (NIHR) Birmingham Biomedical Research Centre. Finally, we thank all individuals at risk of RA from the different countries participating in this study.

Contributors MHvBT, MMtW, LHvT, KR, DvS: substantial contributions to the conception or design of the work or the acquisition, analysis or interpretation of data for the work. All authors contributed to the acquisition of the data. MHvBT, MMtW, KR, DvS: drafting the work or revising it critically for important intellectual content. All authors: final approval of the version to be published; agreement to be accountable for all aspects of the work in ensuring that questions related to the accuracy or integrity of any part of the work are appropriately investigated and resolved.

Funding This project was funded by EULAR (grant number EPI013).

Competing interests None declared.

Patient consent Not required.

Provenance and peer review Not commissioned; externally peer reviewed.

Data sharing statement SPARRA questionnaire: The questionnaire is freely available and has been included in this manuscript. All of the study data can be available if requested.

Open Access This is an Open Access article distributed in accordance with the Creative Commons Attribution Non Commercial (CC BY-NC 4.0) license, which permits others to distribute, remix, adapt, build upon this work non-commercially, and license their derivative works on different terms, provided the original work is properly cited and the use is non-commercial. See: http://creativecommons.org/ licenses/by-nc/4.0/

(c) Article author(s) (or their employer(s) unless otherwise stated in the text of the article) 2018. All rights reserved. No commercial use is permitted unless otherwise expressly granted.

\section{REFERENCES}

1. Nielen MM, van Schaardenburg D, Reesink HW, et al. Specific autoantibodies precede the symptoms of rheumatoid arthritis: a study of serial measurements in blood donors. Arthritis Rheum 2004;50:380-6.

2. Raza K, Gerlag DM. Preclinical inflammatory rheumatic diseases: an overview and relevant nomenclature. Rheum Dis Clin North Am 2014;40:569-80.

3. Kung TN, Bykerk VP. Detecting the earliest signs of rheumatoid arthritis: symptoms and examination. Rheum Dis Clin North Am 2014;40:669-83.

4. Stack RJ, Sahni M, Mallen CD, et al. Symptom complexes at the earliest phases of rheumatoid arthritis: a synthesis of the qualitative literature. Arthritis Care Res 2013;65:1916-26.

5. van Steenbergen HW, van Nies JA, Huizinga TW, et al. Characterising arthralgia in the preclinical phase of rheumatoid arthritis using MRI. Ann Rheum Dis 2015;74:1225-32.
6. van Steenbergen HW, Aletaha D, Beaart-van de Voorde LJ, et al. EULAR definition of arthralgia suspicious for progression to rheumatoid arthritis. Ann Rheum Dis 2017;76:491-6.

7. van Steenbergen HW, van der Helm-van Mil AH. Clinical expertise and its accuracy in differentiating arthralgia patients at risk for rheumatoid arthritis from other patients presenting with joint symptoms. Rheumatology 2016;55:1140-1.

8. Newsum EC, van der Helm-van Mil AH, Kaptein AA. Views on clinically suspect arthralgia: a focus group study. Clin Rheumatol 2016:35:1347-52.

9. Stack RJ, van Tuyl LH, Sloots M, et al. Symptom complexes in patients with seropositive arthralgia and in patients newly diagnosed with rheumatoid arthritis: a qualitative exploration of symptom development. Rheumatology 2014;53:1646-53.

10. van Tuyl LH, Stack RJ, Sloots M, et al. Impact of symptoms on daily life in people at risk of rheumatoid arthritis. Musculoskeletal Care 2016;14:169-73.

11. Lai N-S, Tsai T-Y, Li C-Y, et al. Increased frequency and costs of ambulatory medical care utilization prior to the diagnosis of rheumatoid arthritis: a national population-based study. Arthritis Care Res 2014;66:371-8.

12. Neovius M, Simard JF, Klareskog L, et al. Sick leave and disability pension before and after initiation of antirheumatic therapies in clinical practice. Ann Rheum Dis 2011;70:1407-14

13. Rakieh C, Nam JL, Hunt L, et al. Predicting the development of clinical arthritis in anti-CCP positive individuals with non-specific musculoskeletal symptoms: a prospective observational cohort study. Ann Rheum Dis 2015;74:1659-66.

14. van de Stadt LA, Witte $B I$, Bos $W H$, et al. A prediction rule for the development of arthritis in seropositive arthralgia patients. Ann Rheum Dis 2013;72:1920-6.

15. Burgers LE, van Steenbergen HW, Ten Brinck RM, et al. Differences in the symptomatic phase preceding ACPA-positive and ACPAnegative RA: a longitudinal study in arthralgia during progression to clinical arthritis. Ann Rheum Dis 2017;76:1751-4.

16. Jutley GS, Latif ZP, Raza K. Symptoms in individuals at risk of rheumatoid arthritis. Best Pract Res Clin Rheumatol 2017;31:59-70.

17. Ten Brinck RM, van Steenbergen HW, Mangnus L, et al. Functional limitations in the phase of clinically suspect arthralgia are as serious as in early clinical arthritis; a longitudinal study. RMD Open 2017;3:e000419.

18. World Health Organization. Process of translation and adaptation of instruments. http://www.who.int/substance abuse/research tools/ translation/en/ (accessed 30 Nov 2017).

19. Beaton DE, Bombardier C, Guillemin F, et al. Guidelines for the process of cross-cultural adaptation of self-report measures. Spine 2000;25:3186-91.

20. Bos WH, Wolbink GJ, Boers M, et al. Arthritis development in patients with arthralgia is strongly associated with anti-citrullinated protein antibody status: a prospective cohort study. Ann Rheum Dis 2010;69:490-4

21. Finckh A, Müller RB, Möller B, et al. A novel screening strategy for preclinical rheumatoid arthritis (RA) in first degree relatives of patients with RA. Ann Rheum Dis 2011;70(Suppl 3):282.

22. Hawker GA, Mian S, Kendzerska T, et al. Measures of adult pain: visual analog scale for pain (VAS Pain), Numeric Rating Scale for Pain (NRS Pain), McGill Pain Questionnaire (MPQ), Short-Form McGill Pain Questionnaire (SF-MPQ), Chronic Pain Grade Scale (CPGS), Short Form-36 Bodily Pain Scale (SF-36 BPS), and Measure of Intermittent and Constant Osteoarthritis Pain (ICOAP). Arthritis Care Res 2011;63 Suppl 11(Suppl 11):S240-S252.

23. Hays WL. Statistics. 3rd edn. New York: Holt, Rinehart and Winston, 1981.

24. Kottner J, Streiner DL. The difference between reliability and agreement. J Clin Epidemiol 2011;64-701-2.

25. Landis JR, Koch GG. The measurement of observer agreement for categorical data. Biometrics 1977;33:159-74.

26. Fleiss JL, Cohen J. The equivalence of weighted kappa and the intraclass correlation coefficient as measures of reliability. Educ Psychol Meas 1973;33:613-9.

27. Kolfenbach JR, Deane KD, Derber LA, et al. A prospective approach to investigating the natural history of preclinical rheumatoid arthritis (RA) using first-degree relatives of probands with RA. Arthritis Rheum 2009;61:1735-42.

28. Smolik I, Robinson DB, Bernstein CN, et al. First-degree relatives of patients with rheumatoid arthritis exhibit high prevalence of joint symptoms. J Rheumatol 2013;40:818-24.

29. van Steenbergen HW, Mangnus L, Reijnierse M, et al. Clinical factors, anticitrullinated peptide antibodies and MRI-detected subclinical inflammation in relation to progression from clinically suspect arthralgia to arthritis. Ann Rheum Dis 2016;75:1824-30. 
30. Bell MJ, Tavares R, Guillemin F, et al. Development of a selfadministered early inflammatory arthritis detection tool. $B M C$ Musculoskelet Disord 2010;11:50.

31. Matcham F, Scott IC, Rayner L, et al. The impact of rheumatoid arthritis on quality-of-life assessed using the SF-36: a systematic review and meta-analysis. Semin Arthritis Rheum 2014;44:123-30.

32. Picavet HS, Hoeymans N. Health related quality of life in multiple musculoskeletal diseases: SF-36 and EQ-5D in the DMC3 study. Ann Rheum Dis 2004;63:723-9.

33. Skacelova M, Pavel H, Zuzana H, et al. Relationship between rheumatoid arthritis disease activity assessed with the us7 score and quality of life measured with questionnaires (HAQ, EQ-5D, WPAl). Curr Rheumatol Rev 2017:13:224-230.
34. Gent YY, Voskuyl AE, Kloet RW, et al. Macrophage positron emission tomography imaging as a biomarker for preclinical rheumatoid arthritis: findings of a prospective pilot study. Arthritis Rheum 2012;64:62-6.

35. Krishnamurthy A, Joshua V, Haj Hensvold A, et al. Identification of a novel chemokine-dependent molecular mechanism underlying rheumatoid arthritis-associated autoantibody-mediated bone loss. Ann Rheum Dis 2016;75:721-9.

36. Wigerblad G, Bas DB, Fernades-Cerqueira C, et al. Autoantibodies to citrullinated proteins induce joint pain independent of inflammation via a chemokinedependent mechanism. Ann Rheum Dis 2016;75:730-8. 